\title{
Solving the Ultranonlocality Problem in Time-Dependent Spin-Density-Functional Theory
}

\author{
Z. Qian, A. Constantinescu, and G. Vignale \\ Department of Physics and Astronomy, University of Missouri, Columbia, Missouri 65211
}

(Received 10 September 2002; published 11 February 2003)

\begin{abstract}
It has been known for some time that the exchange-correlation potential in time-dependent densityfunctional theory is an intrinsically nonlocal functional of the density as soon as one goes beyond the adiabatic approximation. In this paper we show that a much more severe nonlocality problem, with a completely different physical origin, plagues the exchange-correlation potentials in time-dependent spin-density functional theory. We show how the use of the spin current density as a basic variable solves this problem, and we provide an explicit local expression for the exchange-correlation fields as functionals of the spin currents.
\end{abstract}

DOI: $10.1103 /$ PhysRevLett.90.066402

PACS numbers: 71.15.Mb, 71.10.Ca, 71.45.Gm

For many years the local density approximation (LDA) has provided the much needed handle on the difficult problem of approximating the density dependence of the exchange-correlation (xc) potential-the single particle potential that incorporates the many-body effects in the Kohn-Sham equation for the ground state density [1]. In LDA, the xc potential $V_{\mathrm{xc}}(\vec{r})$ is simply a function of the local density $n(\vec{r})$. This approximation is not unreasonable as long as the functional derivative of $V_{\mathrm{xc}}(\vec{r})$ with respect to $n\left(\vec{r}^{\prime}\right)$ - the so-called exchange-correlation kernel $f_{\mathrm{xc}}\left(\vec{r}, \vec{r}^{\prime}\right) \equiv\left[\delta V_{\mathrm{xc}}(\vec{r}) / \delta n\left(\vec{r}^{\prime}\right)\right]$-is a sufficiently shortranged function of the distance $\left|\vec{r}-\vec{r}^{\prime}\right|[2]$.

However, much recent work [3-7] has demonstrated that the requirement of short rangedness is not always fulfilled in physical systems, and when this happens the local density approximation is flawed. This does not mean that a local description of exchange and correlation is absolutely impossible, only that such a description cannot be achieved in terms of the particle density.

For example, in the density-functional theory of crystalline insulators it has been found [4-6] that the xc potential has an "ultranonlocal" dependence on the density, due to the fact that the Fourier transform of the xc kernel $f_{\mathrm{xc}}(\vec{k}, \vec{k})$ diverges as $1 / k^{2}$ for $k \rightarrow 0$. But, the ultranonlocality disappears if one reformulates the theory in terms of the electric polarization $\vec{P}(\vec{r})$ and the exchangecorrelation electric field $\vec{E}_{\mathrm{xc}}(\vec{r})$ associated with it.

Another instance of the ultranonlocality problem was discovered in the time-dependent density-functional theory (TDDFT) [8] following the realization that the frequency-dependent LDA [9] fails to satisfy Kohn's theorem $[10,11]$. The pathology was traced to a singularity of the form $\frac{\vec{k} \cdot \vec{k}^{\prime}}{k^{2}}$ in the xc kernel $f_{\mathrm{xc}}\left(\vec{k}, \vec{k}^{\prime}, \omega\right)$ for $k \rightarrow 0$ at finite $\vec{k}^{\prime}$ and $\omega$. The ensuing nonlocality problem was solved by upgrading to time-dependent current-density functional theory (TDCDFT), where the basic variable is the current density, and its conjugate field is a vector potential[3]. TDCDFT has since been applied to the calculation of the optical spectra of solids [12] and the polarizability of long polymer chains [13] with considerable success.

In this Letter we show that the ultranonlocality problem occurs in an aggravated form in the time-dependent spin-density functional theory or, more generally, in the time-dependent DFT of any multi-component system. The novel features of the spin-dependent problem stem from the fact that the xc kernel presents a divergence even in the homogeneous electron liquid. More precisely, it can be shown that the Fourier transform of the spin-dependent exchange-correlation kernel $f_{\mathrm{xc}, \sigma \sigma^{\prime}}\left(r-r^{\prime}, t-t^{\prime}\right) \equiv$ $\left[\delta V_{\mathrm{xc}, \sigma}(\vec{r}, t) / \delta n_{\sigma^{\prime}}\left(\vec{r}^{\prime}, t^{\prime}\right)\right]$ in a homogeneous electron liquid has the long-wavelength expansion

$$
f_{\mathrm{xc}, \sigma \sigma^{\prime}}(k, \omega) \stackrel{k \rightarrow 0}{\longrightarrow} \frac{A(\omega)}{k^{2}} \frac{\sigma \sigma^{\prime} n^{2}}{4 n_{\sigma} n_{\sigma^{\prime}}}+B_{\sigma \sigma^{\prime}}(\omega)+O\left(k^{2}\right),
$$

where $A(\omega)$ and $B_{\sigma \sigma^{\prime}}(\omega)$ are complex functions of frequency, $n_{\sigma}$ is the density of $\sigma$-spin electrons ( $\sigma=+1$ for $\uparrow$-spin and $\sigma=-1$ for $\downarrow$-spin), and $n=n_{\uparrow}+n_{\downarrow}$ is the total density. Since the xc potential created by a small density variation $\delta n_{\sigma}(\vec{k}, \omega)$ is given by the formula

$$
V_{\mathrm{xc}, \sigma}(\vec{k}, \omega)=\sum_{\sigma^{\prime}} f_{\mathrm{xc}, \sigma \sigma^{\prime}}(k, \omega) \delta n_{\sigma^{\prime}}(\vec{k}, \omega),
$$

we see that Eq. (1) rules out the possibility of a local connection between $V_{\mathrm{xc}, \sigma}(\vec{r}, t)$ and $\delta n_{\sigma^{\prime}}\left(\vec{r}^{\prime}, t^{\prime}\right)$.

The existence of the long-wavelength singularity in $f_{\mathrm{xc}, \sigma \sigma^{\prime}}(k, \omega)$ has been known for some time. It was first pointed out by Goodman and Sjölander [14] that the third-moment sum rule for the spin-density response function implies such a singularity. Approximate formulae for $f_{\mathrm{xc},-}(k, \omega)=f_{\mathrm{xc}, \uparrow}(k, \omega)-f_{x c, \uparrow \downarrow}(k, \omega)$ exhibiting the singularity were proposed in [15] and, for imaginary frequencies, in [16]. More recently, D'Amico and Vignale [17] have shown that, at low frequency and finite temperature, the singularity is related to the friction that arises between up- and down-spin currents when they 
have different average velocities (the so-called spin-drag effect).

By contrast, the implications of Eq. (1) for spin-density functional theory have not been explored so far. This is understandable, since the singularity (1) arises only at finite frequency $[A(0)=0]$ and therefore does not affect the static spin DFT. Furthermore, the singularity does not show up in the density response of spin-compensated systems, since the relevant combination of xc kernels, in that case, is $\sum_{\sigma \sigma^{\prime}} n_{\sigma} n_{\sigma^{\prime}} f_{\mathrm{xc}, \sigma \sigma^{\prime}}$, which is nonsingular. It is only in the time-dependent spin DFT [18] that the issue of the singularity becomes really critical not only to the calculation of the spin-response, but even to the calculation of just the density response[19].

In this Letter we propose a resolution of the ultranonlocality problem based on the use of the spin components of the current density $\vec{j}_{\uparrow}(\vec{r}, \omega)$ and $\vec{j}_{\downarrow}(\vec{r}, \omega)$ as basic variables. We provide an explicit expression for the spindependent exchange-correlation field $\vec{E}_{\mathrm{xc}, \sigma}(\vec{r}, \omega)$ as a local linear functional of the currents $\vec{j}_{\sigma}$.

The general method for upgrading from the density to the current-density formulation is described in detail in Ref. [20], so we mention only the essential steps here. We introduce a spin-dependent xc vector potential $\vec{A}_{\mathrm{xc}, \sigma}(\vec{k}, \omega)$ [whose time derivative, $i \omega \vec{A}_{\mathrm{xc}, \sigma}(\vec{k}, \omega)=$ $\vec{E}_{\mathrm{xc}}(\vec{k}, \omega)$, is the xc electric field], and notice that this is linearly related to the currents in the following manner

$$
A_{\mathrm{xc}, \sigma}^{\alpha}(\vec{k}, \omega)=\frac{k^{2}}{\omega^{2}} \sum_{\sigma^{\prime}} f_{\mathrm{xc}, \sigma \sigma^{\prime}}^{\alpha}(\vec{k}, \omega) j_{\sigma^{\prime}}^{\alpha}(\vec{k}, \omega),
$$

where the superscript $\alpha$ denotes the longitudinal $(\alpha=L)$ or transverse $(\alpha=T)$ component of a vector relative to the direction of $\vec{k}$. It is not difficult to see that the longitudinal xc kernel defined in this manner coincides with the xc kernel of Eq. (1). The extra factor $k^{2} / \omega^{2}$ in Eq. (3) exactly cancels the small- $k$ singularity of $f_{\mathrm{xc}}$, and leads to a theory that admits a local approximation. The imaginary part of the current xc kernel $f_{\mathrm{xc}, \sigma \sigma^{\prime}}^{\alpha}(k, \omega)$ is expressed in terms of a causal response function as follows:

$$
\operatorname{Im} f_{\mathrm{xc}, \sigma \sigma^{\prime}}^{\alpha}(k, \omega)=\frac{1}{V n_{\sigma} n_{\sigma^{\prime}} k^{2}} \operatorname{Im}\left\langle\left\langle\hat{F}_{\sigma}^{\alpha}(\vec{k}) ; \hat{F}_{\sigma^{\prime}}^{\alpha}(-\vec{k})\right\rangle\right\rangle_{\omega},
$$

where $\langle\langle\hat{A} ; \hat{B}\rangle\rangle_{\omega} \equiv-\frac{i}{\hbar} \int_{0}^{\infty}\langle[\hat{A}(t), \hat{B}]\rangle e^{i \omega t} d t$ is the linear response function associated with the operators $\hat{A}$ and $\hat{B} ; \hat{F}_{\sigma}^{\alpha}(\vec{k})=-\frac{i m}{\hbar}\left[\hat{H}, \hat{j}_{\sigma}^{\alpha}(\vec{k})\right]$ is the time derivative of the Fourier transform of the current-density operator $\hat{\vec{j}}_{\sigma}(\vec{k})$, $\hat{H}$ is the Hamiltonian, and $V$ is the volume.

Once the imaginary part of $f_{\mathrm{xc}, \sigma \sigma^{\prime}}^{\alpha}(k, \omega)$ is known, its real part is determined by the Kramers-Krönig dispersion relation

$$
\begin{aligned}
\operatorname{Re} f_{\mathrm{xc}, \sigma \sigma^{\prime}}^{\alpha}(k, \omega)= & f_{\mathrm{xc}, \sigma \sigma^{\prime}}^{\alpha}(k, \infty) \\
& -\frac{2}{\pi} \mathcal{P} \int_{0}^{\infty} d \omega^{\prime} \frac{\omega^{\prime} \operatorname{Im} f_{\mathrm{xc}, \sigma \sigma^{\prime}}^{\alpha}\left(k, \omega^{\prime}\right)}{\omega^{2}-\omega^{\prime 2}},
\end{aligned}
$$

where $\mathcal{P}$ denotes the principal part integral, and the infinite frequency limit of $f_{\mathrm{xc}, \sigma \sigma^{\prime}}$ is determined by the third moment sum rule. In a three-dimensional electron liquid, this sum rule gives

$$
\begin{aligned}
f_{\mathrm{xc}, \sigma \sigma^{\prime}}^{\alpha}(k, \infty) \stackrel{k \rightarrow 0}{\longrightarrow} & -\frac{4 \pi e^{2}}{3 k^{2}} \frac{n_{\uparrow} n_{\downarrow}}{n_{\sigma} n_{\sigma^{\prime}}}\left[g_{\uparrow \downarrow}(0)-1\right] \sigma \sigma^{\prime} \\
& +a^{\alpha} \frac{t_{c \sigma}}{n_{\sigma}} \delta_{\sigma \sigma^{\prime}}+\frac{b^{\alpha}}{2} \int d \vec{r} \frac{e^{2}}{r}\left[g_{\sigma \sigma^{\prime}}(r)-1\right],
\end{aligned}
$$

where $a^{L}=2, a^{T}=2 / 3, b^{L}=4 / 15$, and $b^{T}=-2 / 15$. Here $g_{\sigma \sigma^{\prime}}(r)$ is the spin-resolved pair correlation function and $t_{c \sigma}$ is the average correlation kinetic energy of the $\sigma$-spin component. Note that the result for the longitudinal case was first obtained in Ref. [14].

It is evident from the above equations that both the longitudinal and the transverse kernels exhibit $1 / k^{2} \sin$ gularities, which are "cured" by the $k^{2} / \omega^{2}$ factor of Eq. (3). In particular, substituting the small- $k$ expansion $\hat{F}_{\sigma}^{\alpha}(\vec{k})=\hat{F}_{\sigma}^{\alpha}(0)+O(\vec{k})$ in Eq. (4), where $\hat{F}_{\sigma}^{\alpha}(0)$ is the operator of the total force acting on $\sigma$-spin electrons, and noting that terms of first order in $\vec{k}$ vanish by inversion symmetry, we see that the xc kernels have the small- $k$ expansion

$$
f_{\mathrm{xc}, \sigma \sigma^{\prime}}^{\alpha}(k, \omega) \stackrel{k \rightarrow 0}{\longrightarrow} \frac{A(\omega)}{k^{2}} \frac{\sigma \sigma^{\prime} n^{2}}{4 n_{\sigma} n_{\sigma^{\prime}}}+B_{\sigma \sigma^{\prime}}^{\alpha}(\omega)+O\left(k^{2}\right),
$$

where

$$
\operatorname{Im} A(\omega)=-\frac{4}{V n^{2}} \operatorname{Im}\left\langle\left\langle\hat{F}_{\uparrow}^{\alpha} ; \hat{F}_{\downarrow}^{\alpha}\right\rangle\right\rangle_{\omega}
$$

and

$$
\begin{aligned}
\operatorname{Re} A(\omega)= & -\frac{16 \pi e^{2}}{3}\left[g_{\uparrow \downarrow}(0)-1\right] \\
& -\frac{2}{\pi} \mathcal{P} \int_{0}^{\infty} d \omega^{\prime} \frac{\omega^{\prime} \operatorname{Im} A\left(\omega^{\prime}\right)}{\omega^{2}-\omega^{\prime 2}} .
\end{aligned}
$$

The factor $\sigma \sigma^{\prime}$ in Eq. (7) arises from the fact that the total force $\hat{F}_{\uparrow}+\hat{F}_{\downarrow}$ vanishes, due to translational invariance, so that $\left\langle\left\langle\hat{F}_{\sigma} ; \hat{F}_{\sigma^{\prime}}\right\rangle\right\rangle_{\omega}=-\sigma \sigma^{\prime}\left\langle\left\langle\hat{F}_{\uparrow} ; \hat{F}_{\downarrow}\right\rangle\right\rangle_{\omega}$. Notice also that $A(\omega)$ is independent of the direction $\alpha$-longitudinal or transverse. The microscopic expression for $B_{\sigma \sigma^{\prime}}^{\alpha}$ is more complicated: a simple approximation for this quantity will be presented below.

Substituting the expansion (7) into Eq. (3), calculations similar to those described in [20] lead us to the following local approximation for the xc field in terms of the spin currents 


$$
\begin{aligned}
-e \vec{E}_{\mathrm{xc}, \sigma}(\omega)= & -\vec{\nabla} V_{\mathrm{xc}, \sigma}^{\mathrm{LDA}}+\frac{1}{n_{\sigma}} \vec{\nabla} \cdot \overleftrightarrow{\sigma}_{\mathrm{xc}, \sigma}(\omega) \\
& +\frac{i n^{2} A(\omega)}{4 \omega} \sum_{\sigma^{\prime}} \frac{\sigma \sigma^{\prime}}{n_{\sigma} n_{\sigma^{\prime}}} \vec{j}_{\sigma^{\prime}}
\end{aligned}
$$

Here the $\vec{r}$ dependence has been left implicit, and the xc stress tensor $\vec{\sigma}_{\text {xc }}(\omega)$, as well as $A(\omega)$, is a function of the local spin densities, as discussed below.

Equation (10) is the central result of this paper. The first two terms on the right are well known: they are, respectively, the adiabatic LDA contribution and the viscoelastic force term, where the stress tensor $\sigma_{\mathrm{xc}, \sigma}(\omega)$ is related to $B_{\mathrm{xc}, \sigma \sigma^{\prime}}$ by obvious extensions of the formulas reported in [20]. The expression for the xc stress tensor is

$$
\begin{aligned}
\sigma_{\mathrm{xc}, \sigma, i j}=\sum_{\sigma^{\prime}}[ & \eta_{x c, \sigma \sigma^{\prime}}\left(\frac{\partial u_{\sigma^{\prime}, i}}{\partial r_{j}}+\frac{\partial u_{\sigma^{\prime}, j}}{\partial r_{i}}-\frac{2}{3} \vec{\nabla} \cdot \vec{u}_{\sigma^{\prime}} \delta_{i j}\right) \\
& \left.+\zeta_{\mathrm{xc}, \sigma \sigma^{\prime}} \vec{\nabla} \cdot \vec{u}_{\sigma^{\prime}} \delta_{i j}\right],
\end{aligned}
$$

where $\vec{u}_{\sigma}=\vec{j}_{\sigma} / n_{\sigma}$, and

$$
\begin{gathered}
\eta_{\mathrm{xc}, \sigma \sigma^{\prime}}=-\frac{n_{\sigma} n_{\sigma^{\prime}}}{i \omega} B_{\sigma \sigma^{\prime}}^{T}(\omega), \\
\zeta_{\mathrm{xc}, \sigma \sigma^{\prime}}=-\frac{n_{\sigma} n_{\sigma^{\prime}}}{i \omega}\left[B_{\sigma \sigma^{\prime}}^{L}(\omega)-\frac{4}{3} B_{\sigma \sigma^{\prime}}^{T}(\omega)-\epsilon_{\mathrm{xc}, \sigma \sigma^{\prime}}^{\prime \prime}\right]
\end{gathered}
$$

where $\epsilon_{\mathrm{xc}, \sigma \sigma^{\prime}}^{\prime \prime}=\left(\partial^{2} \epsilon_{\mathrm{xc}} / \partial n_{\sigma} \partial n_{\sigma^{\prime}}\right)$. The last term in Eq. (10) is new, and comes directly from the $1 / k^{2}$ singularity of Eq. (7). The essential feature of the new term is that it produces damping of the spin-current proportional to the relative velocity between up- and down-spin electrons. This makes it readily distinguishable from the usual viscous friction contained in the second term, which is proportional to the derivatives of the velocity field. The physical reason for the difference is that, whenever up and down-spin currents travel with different average velocities, they exert friction on each other: the "spin drag coefficient" is $\gamma(\omega)=\left[i n^{3} A(\omega) / 4 \omega m n_{\uparrow} n_{\downarrow}\right]$. Of course, like all the quantities considered here, $\gamma(\omega)$ is complex and frequency dependent, and, in the limit of zero frequency, its real part can be shown to be related to the spin

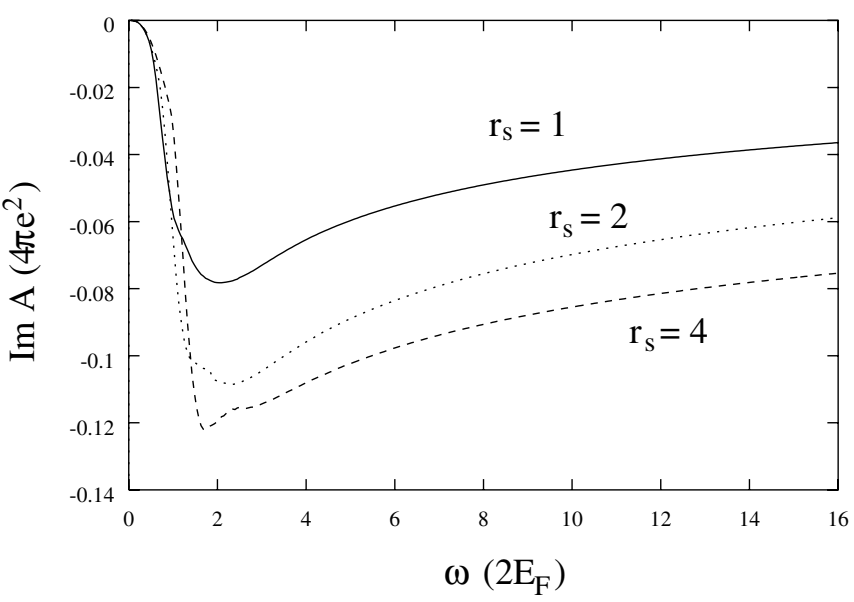

FIG. 1. Imaginary part of $A(\omega)$ evaluated from Eq. (14) with the correction factor given in Eq. (16). The values of $a\left(r_{s}, 0\right)$ are $1.92,3.36$, and 7.49 at $r_{s}=1,2$, and 4 , respectively.

diffusion constant $D_{s}$ by the Einstein relation $D_{s}=$ $\left[n / m \chi_{s} \gamma(0)\right]$, where $\chi_{s}$ is the static, macroscopic spin susceptibility.

Unfortunately, an exact calculation of $A(\omega)$ from the microscopic expressions (8) and (9) is beyond the reach of present-day many-body techniques. However, we can obtain a rather good approximation with the help of the following exact results: (i) For $\omega \rightarrow 0, \operatorname{Im} A(\omega) \propto \omega^{3}$ and $\operatorname{Re} A(\omega) \propto \omega^{2}$; (ii) For large $\omega, \operatorname{Im} A(\omega) \rightarrow-\left(16 \pi e^{2} / 3\right) \times$ $\left(n_{\uparrow} n_{\downarrow} / n^{2}\right)\left(\alpha r_{s} / \sqrt{\bar{\omega}}\right)\left[1 /(1+\zeta)^{1 / 3}\right] \quad$ and $\quad \operatorname{Re} A(\omega) \rightarrow$ $-\left(16 \pi e^{2} / 3\right)\left(n_{\uparrow} n_{\downarrow} / n^{2}\right)\left[g_{\uparrow \downarrow}(0)-1\right]$. Here $\bar{\omega}=\left(\omega / 2 E_{F \uparrow}\right)$, where $E_{F \uparrow}$ is the Fermi energy for majority spin electrons and $\zeta=\left(n_{\uparrow}-n_{\downarrow}\right) / n$ measures the degree of spin polarization, and $\alpha=(4 / 9 \pi)^{1 / 3}$ [21]. Note that $g_{\uparrow \downarrow}(0)$ is accurately known from the work of Gori-Giorgi and Perdew [22]. The high and low frequency limits of $\operatorname{Re} A(\omega)$ are both obtained from the third-moment sum rule. In particular, the vanishing of $\operatorname{Re} A(0)$ follows from the fact that $\frac{2}{\pi} \int_{0}^{\infty}\left[\operatorname{Im} A\left(\omega^{\prime}\right) / \omega^{\prime}\right]$ is equal to (minus) the first moment of the current-current response function, which, by gauge invariance and the continuity equation, coincides with the third-moment of the density-density response function, i.e., $-A(\infty)$.

The $\omega^{3}$ behavior of $\operatorname{Im} A(\omega)$ at low frequency is easily obtained from the approximate zero-temperature formula [17]

$$
\operatorname{Im} A(\omega) \simeq-\frac{4}{3 n^{2} V} \sum_{\vec{q}} v_{\vec{q}}^{2} q^{2} \int_{0}^{\omega} \frac{d \omega^{\prime}}{\pi}\left[\operatorname{Im} \chi_{\Uparrow \uparrow}\left(q, \omega-\omega^{\prime}\right) \operatorname{Im} \chi_{\Downarrow \downarrow}\left(q, \omega^{\prime}\right)-\operatorname{Im} \chi_{\uparrow \downarrow}\left(q, \omega-\omega^{\prime}\right) \operatorname{Im} \chi_{\Downarrow \uparrow}\left(q, \omega^{\prime}\right)\right],
$$

which is exact in the limits of high density and high frequency. Here $v_{\vec{q}}=\frac{4 \pi e^{2}}{q^{2}}$ and $\chi_{\sigma \sigma^{\prime}}(q, \omega)$ are the spindensity response functions of the homogeneous liquid.

We have evaluated $\chi_{\sigma \sigma^{\prime}}$ in the generalized random phase approximation

$$
\chi_{\sigma \sigma^{\prime}}^{-1}(q, \omega)=\left[\chi_{\sigma}^{(0)}\right]^{-1}(q, \omega) \delta_{\sigma \sigma^{\prime}}-v_{\vec{q}}\left[1-G_{\sigma \sigma^{\prime}}(q)\right],
$$

where $\chi_{\sigma}^{(0)}(q, \omega)$ is the Lindhard function and $G_{\sigma \sigma^{\prime}}(q)$ are local field corrections [23]. At typical metallic densities we multiply $\operatorname{Im} A(\omega)$ by an empirical factor

$$
g(\omega)=\frac{1+\sqrt{\bar{\omega}}}{a\left(r_{s}, \zeta\right)+\sqrt{\bar{\omega}}}
$$

designed to satisfy the condition $\operatorname{Re} A(0)=0$ without 


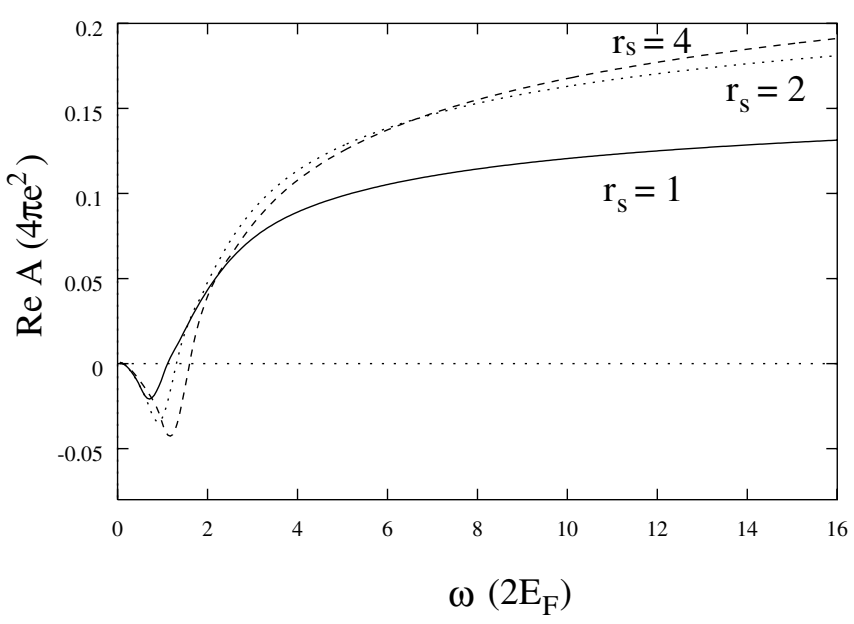

FIG. 2. Real part of $A(\omega)$ obtained from Eq. (9).

altering the high-frequency behavior. Notice that $a\left(r_{s}, \zeta\right) \rightarrow 1$ for $r_{s} \rightarrow 0$. The results evaluated with this procedure are shown in Figs. 1 and 2.

Finally, we briefly remark on the calculation of the regular part of the xc kernel. The spin symmetric combinations $B_{+}^{\alpha} \equiv B_{\Uparrow}^{\alpha}+B_{\uparrow \downarrow}^{\alpha}$ with $\alpha=L$ or $T$ have been approximately calculated, in the paramagnetic state $(\zeta=0)$, by two of us [24]. For the antisymmetric combinations $B_{-}^{\alpha} \equiv B_{\Uparrow}^{\alpha}-B_{\uparrow \downarrow}^{\alpha}$ a provisional solution is offered by the time-honored Gross-Kohn interpolation formula [9], with parameters suitably tuned to satisfy the exact identity $\lim _{\omega \rightarrow 0}\left\{\left[B_{\sigma \sigma^{\prime}}^{L}(\omega)-\frac{4}{3} B_{\sigma \sigma^{\prime}}^{T}(\omega)-\epsilon_{\mathrm{xc}, \sigma \sigma^{\prime}}^{\prime \prime}\right] / \omega\right\}=0$ [25], the third-moment sum rule, and the limiting form $B_{-}^{\alpha}(\omega) \rightarrow \omega^{-3 / 2}$ for $\omega \rightarrow \infty$. Finally, for general polarization, we propose to use the formula $B_{\sigma \sigma^{\prime}}^{\alpha}(\zeta)=$ $P_{\sigma \sigma^{\prime}}(\zeta) B_{\sigma \sigma^{\prime}}^{\alpha}(\zeta=0) \quad$ where $\quad P_{\sigma \sigma^{\prime}}(\zeta)=\left[\epsilon_{\mathrm{xc}, \sigma \sigma^{\prime}}^{\prime \prime}(\zeta) /\right.$ $\left.\epsilon_{\mathrm{xc}, \sigma \sigma^{\prime}}^{\prime \prime}(0)\right]$, so that the above conditions are satisfied for all $\zeta$. This completes the construction of the input for Eq. (10). We hope that the new expression for the spincurrent dependent xc field will stimulate applications of CDFT to the calculation of spin excitations in spinpolarized systems.

This work was supported by NSF Grant No. DMR0074959. We acknowledge useful discussions with Carsten Ullrich, Paul de Boeij, and Robert van Leeuwen.

[1] R. M. Dreizler and E. K. U. Gross, Density Functional Theory (Springer-Verlag, Berlin, 1990).
[2] More precisely, the Fourier transform of the xc kernel $f_{\mathrm{xc}}\left(\vec{k}, \vec{k}^{\prime}\right)$ must have a finite limit for $\vec{k}$ and/or $\vec{k}^{\prime}$ tending to zero.

[3] G. Vignale and W. Kohn, Phys. Rev. Lett. 77, 2037 (1996); G. Vignale, C. A. Ullrich, and S. Conti, Phys. Rev. Lett. 79, 4878 (1997).

[4] X. Gonze, Ph. Ghosez, and R.W. Godby, Phys. Rev. Lett. 74, 4035 (1995); 78, 294 (1997); Ph. Ghosez, X. Gonze, and R.W. Godby, Phys. Rev. B 56, 12811 (1997).

[5] R. M. Martin and G. Ortiz, Phys. Rev. B 56, 1124 (1997); G. Ortiz, I. Souza, and R. M. Martin, Phys. Rev. Lett. 80, 353 (1998).

[6] D. Vanderbilt, Phys. Rev. Lett. 79, 3966 (1997).

[7] I.V. Tokatly and O. Pankratov, Phys. Rev. Lett. 86, 2078 (2001).

[8] E. K. U. Gross, J. F. Dobson, and M. Petersilka, in Density Functional Theory II, edited by R. F. Nalewajski, Topics in Current Chemistry (Springer, Berlin, 1996), Vol. 181, p. 81.

[9] E. K. U. Gross and W. Kohn, Phys. Rev. Lett. 55, 2850 (1985); 57, 923(E) (1986).

[10] J. F. Dobson, Phys. Rev. Lett. 73, 2244 (1994).

[11] W. Kohn, Phys. Rev. 123, 1242 (1961).

[12] P. L. de Boeij, F. Kootstra, J. A. Berger, R. van Leeuwen, and J. G. Snijders, J. Chem. Phys. 115, 1995 (2001).

[13] M. van Faassen, P. L. de Boeij, R. van Leeuwen, J. A. Berger, and J. G. Snijders, Phys. Rev. Lett. 88, 186401 (2002).

[14] B. Goodman and A. Sjölander, Phys. Rev. 8, 200 (1973).

[15] K. L. Liu, Can. J. Phys. 69, 573 (1991).

[16] C. F. Richardson and N.W. Ashcroft, Phys. Rev. B 50, 8170 (1994).

[17] I. D'Amico and G. Vignale, Phys. Rev. B 62, 4853 (2000).

[18] K. L. Liu and S. H. Vosko, Can. J. Phys. 67, 1015 (1989).

[19] Remarkably, the singularity does not affect the transverse spin-response discussed in Z. Qian and G. Vignale, Phys. Rev. Lett. 88, 056404 (2002).

[20] C. A. Ullrich and G. Vignale, Phys. Rev. B 65, 245102 (2002); S. Conti and G. Vignale, Phys. Rev. B 60, 7966 (1999).

[21] The large $\omega$ limit of $\operatorname{Im} A(\omega)$ at $\zeta=0$ was also obtained by Liu [15]. However, his assumption that $\operatorname{Im} A(\omega) \propto \omega$ for $\omega \rightarrow 0$ is incorrect.

[22] P. Gori-Giorgi and J. P. Perdew, Phys. Rev. B 64, 155102 (2001).

[23] N. Iwamoto and D. Pines, Phys. Rev. B 29, 3924 (1984).

[24] Z. Qian and G. Vignale, Phys. Rev. B 65, 235121 (2002).

[25] This is the spin-dependent generalization of an exact identity first established in the spinless case (see Ref. [20]). 